\title{
Inhaled frusemide and exercise-induced bronchoconstriction in children with asthma
}

Paul Munyard, K Fan Chung, Andrew Bush

\begin{abstract}
Background - Nebulised frusemide has been shown to be protective against bronchoconstricting stimuli in adult asthmatic subjects and against cold air challenge in children. Animal studies suggest that inhaled frusemide may be more effective in the young.

Methods - A double blind placebo, controlled, crossover study on the effect on exercise of pretreatment with frusemide $(20 \mathrm{mg})$ from a metered dose inhaler via a large volume spacer (Volumatic) was performed in 12 asthmatic children. Exercise testing consisted of eight minutes of running on a treadmill in an environmentally controlled laboratory.

Results - Deterioration in lung function was less after frusemide than after the placebo exercise tests. The mean ( $95 \%$ CI) maximum percentage falls in forced expiratory volume in one second $\left(\mathrm{FEV}_{1}\right)$ were $14.4 \%(7 \cdot 7$ to $21 \cdot 0)$ for placebo and $5 \cdot 7 \%$ (2.3 to $9 \cdot 0)$ for frusemide.

Conclusions - Inhaled frusemide via a metered dose inhaler reduces exerciseinduced bronchoconstriction in children. (Thorax 1995;50:677-679)
\end{abstract}

Keywords: asthma, children, frusemide.

Nebulised (but not intravenous or oral) frusemide has been shown in adult asthmatic subjects to be protective against bronchoconstriction induced by allergen, ${ }^{1}$ exercise, ${ }^{2}$ sodium metabisulphite. ${ }^{4}$ Nebulised frusemide also protects against cold air-induced bronchoconstriction in children with mild to moderate asthma. ${ }^{5}$

Animal studies suggest a more pronounced effect and a longer duration of action of frusemide in newborn than in adult guinea pigs ${ }^{6}$; there is therefore a need for studies in children to establish its protective profile.

Department of Thoracic Medicine K F Chung

National Heart and Lung Institute, London SW3 6LY, UK

Reprint requests to: Dr A Bush.

Received 21 July 1994 Returned to authors 28 September 1994 Revised version received 13 December 1994 Accepted for publication 28 February 1995

\section{SUBJECTS}

Methods

Twelve patients (eight boys) aged 7-14 years (mean 10.6 years) were recruited from the asthma clinic of the Royal Brompton Hospital. Patients were recruited if they complained of exercise-induced asthma and agreed to participate in the study. Asthma was diagnosed on the basis of both a clinical history of intermittent respiratory symptoms and either a ultrasonically nebulised water, ${ }^{3}$ and nebulised

peak flow lability of $20 \%$ or an improvement in forced expiratory flow in one second $\left(\mathrm{FEV}_{1}\right)$ of $15 \%$ with inhaled $\beta_{2}$ agonists. All patients were on inhaled prophylactic therapy (11 budesonide, dosage range $200-1600 \mu \mathrm{g}$ per day; one disodium cromoglycate). No patient was taking oral steroids, theophyllines, long acting $\beta_{2}$ agonists, or antihistamines at the time of the study. All patients were free from respiratory infection for at least one month before the study. Written informed consent was obtained from their parents, verbal consent from the child, and the protocol was approved by the hospital ethics committee.

\section{PROTOCOL}

We conducted a double blind placebo controlled crossover trial with standardised exercise testing. Before testing disodium cromoglycate was withheld for 24 hours, inhaled $\beta_{2}$ agonists for six hours, and inhaled steroid therapy was unchanged. Three standardised exercise tests were undertaken at the same time of day, all within a period of seven days as previously recommended. ${ }^{7}$ Frusemide $(20 \mathrm{mg})$ or matched placebo (Hoechst UK Ltd) was administered by metered dose inhaler ( $5 \mathrm{mg} / \mathrm{puff}$ ) through a Volumatic $750 \mathrm{ml}$ spacer (Allen \& Hanburys) in random order.

Flow-volume loops were performed with a portable compact spirometer (Vitalograph $\mathrm{UK}$ ), selecting the loop with the best $\mathrm{FEV}_{1}$ from three technically satisfactory measurements. Spirometric tests were performed at baseline (before frusemide or placebo administration), at four minutes during the exercise, at the end of exercise, and at $2,4,6,8$, $10,15,20$, and 30 minutes after exercise.

\section{EXERCISE TESTING}

The exercise testing consisted of eight minutes of running on a treadmill with increasing speed and gradient to a maximum of 4.5 miles per hour and $15 \%$ incline. Subjects were exercised up to $85 \%$ of their age predicted maximal heart rate. Tests were performed in an air conditioned laboratory under controlled and identical environmental conditions.

\section{STATISTICAL ANALYSIS}

The results were normalised by dividing by the baseline measurement on that particular day. The results from the three exercise tests were analysed by two way analysis of variance using the statistics package SPSS. 
Baseline FEV (actual value (l/min), percentage predicted, and means) on control, placebo, and frusemide days

\begin{tabular}{llll}
\hline Patient & Control & Placebo & Frusemide \\
\hline 1 & $1.91(72 \%)$ & $1.87(70 \%)$ & $1.86(70 \%)$ \\
2 & $1.98(90 \%)$ & $1.93(88 \%)$ & $1.88(85 \%)$ \\
3 & $1.93(72 \%)$ & $1.93(72 \%)$ & $1.83(69 \%)$ \\
4 & $3.22(94 \%)$ & $3.15(92 \%)$ & $3.23(95 \%)$ \\
5 & $1.71(98 \%)$ & $1.73(99 \%)$ & $1.73(99 \%)$ \\
6 & $2.42(98 \%)$ & $2.37(96 \%)$ & $2.52(102 \%)$ \\
7 & $1.35(96 \%)$ & $1.38(99 \%)$ & $1.34(96 \%)$ \\
8 & $1.62(87 \%)$ & $1.62(87 \%)$ & $1.58(84 \%)$ \\
9 & $1.34(82 \%)$ & $1.34(82 \%)$ & $1.32(81 \%)$ \\
10 & $1.41(89 \%)$ & $1.25(78 \%)$ & $1.27(79 \%)$ \\
11 & $2.48(83 \%)$ & $2.64(88 \%)$ & $2.72(90 \%)$ \\
12 & $2.57(82 \%)$ & $2.48(80 \%)$ & $2.39(77 \%)$ \\
Mean & $2.0(86.9 \%)$ & $2.0(85.9 \%)$ & $2.0(85.6 \%)$ \\
\hline
\end{tabular}

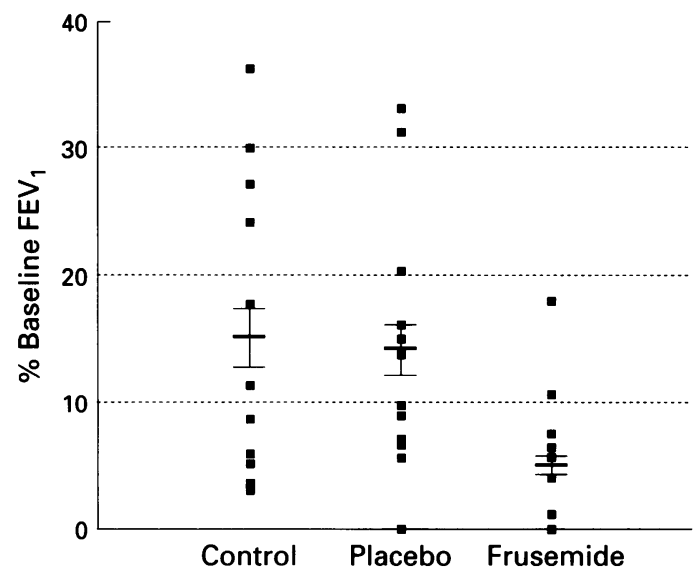

Figure 1 Maximal percentage fall from baseline in $F E V_{1}$ (actual values, means, and standard error of means (error bars)).

\section{Results}

There were no significant differences in baseline $\mathrm{FEV}_{1}$, peak expiratory flow rate (PEFR), or forced mid expiratory volume $\left(\mathrm{FEF}_{25-75}\right)$ between the three study days. The mean (SE) percentage predicted values for baseline $\mathrm{FEV}_{1}$ were: control $86.9 \%(2.6 \%)$, placebo $85.9 \%$ $(2 \cdot 8 \%)$, and frusemide $85 \cdot 6 \%(3 \cdot 2 \%)$. The

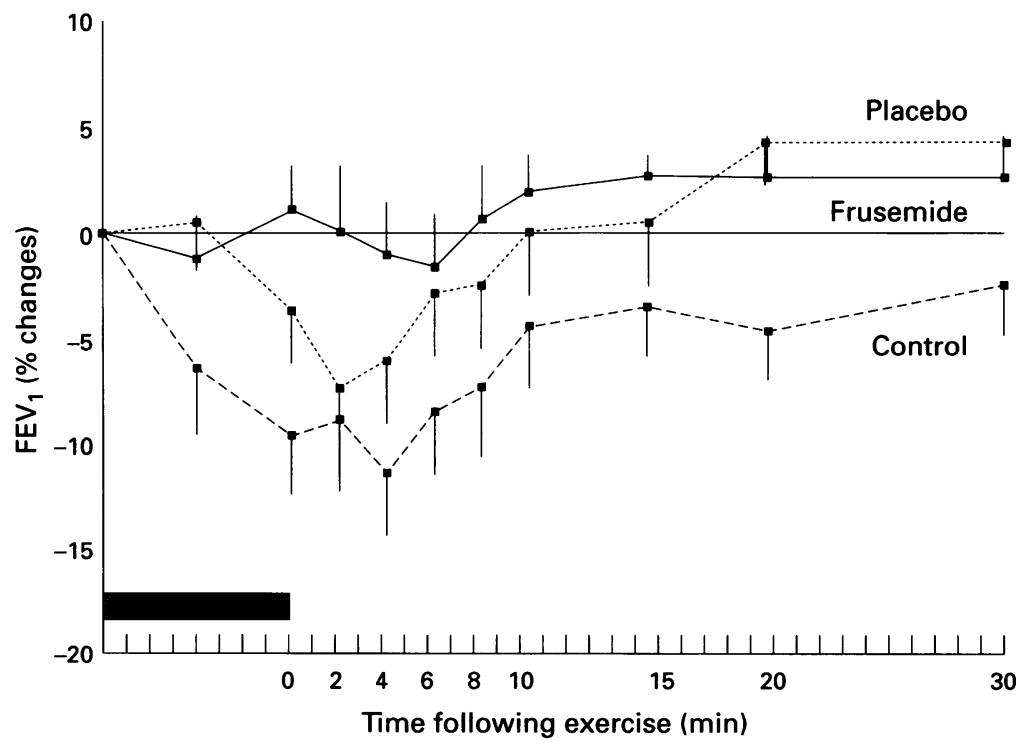

Figure 2 Percentage change from baseline in FEV over the time of the study for the three exercise tests.
$\mathrm{FEV}_{1}$ values (actual values, percentage predicted, and means) are shown in the table.

The mean maximal (SE) percentage falls in $\mathrm{FEV}_{1}$ are shown in fig 1 . The mean $(95 \%$ confidence intervals) maximal percentage falls in $\mathrm{FEV}_{1}$ were: control $15.4 \%$ ( 7.5 to 23.3$)$, placebo $14 \cdot 4 \%(7 \cdot 7$ to $21 \cdot 0)$, frusemide $5 \cdot 7 \%$ $(2 \cdot 3$ to $9 \cdot 0)$. The results for the control and placebo days were not statistically different, while the results for the frusemide day were statistically different from both the control and placebo days $(p=0.005)$. Thus, the degree of protection afforded by frusemide (calculated as the differences in changes of lung function after placebo and frusemide expressed as percentage of the change after placebo) was $60 \%$.

The mean $(95 \%$ confidence intervals) maximal percentage falls in PEFR were: control $18 \cdot 4 \%(13 \cdot 2$ to $23 \cdot 6)$, placebo $21 \cdot 1 \%(12 \cdot 0$ to $30 \cdot 2)$, frusemide $12 \cdot 5 \%(7 \cdot 7$ to $17 \cdot 2)$. The differences with frusemide treatment did not reach statistical significance. There was no statistically significant difference between mean maximal percentage falls in $\mathrm{FEF}_{25-75}$.

Figure 2 shows the percentage change from baseline in $\mathrm{FEV}_{1}$ over the time of the study for the three exercise tests.

\section{Discussion}

We have shown that frusemide, delivered by a metered dose inhaler and spacer, is protective against exercise-induced bronchoconstriction in asthmatic children on prophylactic therapy. This is the first use of frusemide by this route in children. A previous study in children ${ }^{5}$ found a similar degree of protection in $\mathrm{FEV}_{1}$ (63\%) as our study, but the patient characteristics, stimulus, dose, and route of drug administration were different. We did not study mildly asthmatic children, and further studies are needed to compare $\beta_{2}$ agonists and frusemide in this situation. The placebo effect gave minor, statistically insignificant protection in $\mathrm{FEV}_{1}$ and $\mathrm{FEF}_{25-75}$. The degree of protection afforded to $\mathrm{FEV}_{1}$ in adult studies has been reported at $67 \%$ and $62 \% .^{28}$ The results from our study were similar, with no greater efficacy of frusemide in children; however, there were important differences between these studies, including patient characteristics.

We encountered no adverse effects with frusemide administration. The exact mechanism of action of frusemide is yet to be determined. Further studies addressing the duration of this protective effect, its long term safety, and more directly comparable studies in adults and children need to be undertaken.

The authors would like to thank Hoechst for providing the metered dose inhalers and for financial support.

Bianco S, Pieroni M, Refini RM, Rottoli L, Sestini P. Protective effect of inhaled furosemide on allergen induced early and late asthmatic reactions. $N$ Engl f Med 1989;351: 1069-73.

2 Bianco S, Vaghi A, Roubushi M, Pasargiklian M. Prevention of exercise induced bronchoconstriction by inhaled furosemide. Lancet 1988;ii:252-5.

3 Robuschi M, Gambaro G, Spagnotto S, Vaghi A, Bianco $S$. Inhaled furosemide is highly effective in preventing ultrasonically nebulised water bronchoconstriction. Pulm ultrasonically nebulised wate

4 Nichol GM, Alton EW, Nix A, Geddes DM, Chung KF, 
Barnes PJ. Effect of inhaled furosemide on metabisulfite and methacholine induced bronchoconstriction and nasal and methacholine induced bronchoconstriction and nasal
potential difference in asthmatic subjects. Am Rev Respir

5 Seidenberg J, Dehning J, von der Hart H. Inhaled frusemide against cold air induced bronchoconstriction in asthmatic children. Arch Dis Child 1992;67:214-7.

6 Stevens EL, Vyehara CFT, Southgate WM, Nakamura KT.
Furosemide differently relaxes airway and vascular smooth muscle in fetal, newborn and adult guinea-pigs. Am Rev Respir Dis 1992;146:1192-7.

7 Silverman M, Anderson SD. Standardization of exercise tests in asthmatic children. Arch Dis Child 1972;47:882-9.

8 Pavord ID, Wisniewski A, Tattersfield AE. Inhaled frusemide and exercise induced asthma: evidence for a role of inhibitory prostanoids. Thorax 1992;47:797-800.

\title{
Salmeterol inhaler using a non-chlorinated propellant, HFA134a: systemic pharmacodynamic activity in healthy volunteers
}

\author{
S M Kirby, J Smith, G P Ventresca
}

\begin{abstract}
Background - Metered dose inhalers for the treatment of asthma use chlorofluorocarbons as propellants. These face an international ban due to their effect on the ozone layer. Salmeterol has been reformulated using the non-chlorinated propellant Glaxo inhalation grade HFA134a.

Methods - The safety, tolerability and systemic pharmacodynamic activity of the salmeterol/HFA134a inhaler, the current salmeterol inhaler, and placebo (HFA134a) were compared in 12 healthy volunteers in a double blind, randomised crossover study using a cumulative dosing design. Results - Safety and tolerability were similar and the response was related to the dose over the range used $(50-400 \mu \mathrm{g})$ with both salmeterol inhalers. The salmeterol/ HFA134a inhaler showed no differences from the current inhaler for pulse rate, blood pressure, tremor, $\mathbf{Q T}_{\mathrm{c}}$ interval, and plasma glucose levels. The salmeterol/ HFA134a inhaler had significantly less effect on plasma potassium levels.

Conclusions - In healthy volunteers the salmeterol/HFA134a inhaler is at least as safe and well tolerated as the current salmeterol inhaler, and has similar systemic pharmacodynamic activity.

(Thorax 1995;50:679-681)
\end{abstract}

Keywords: HFA134a, salmeterol, metered dose inhalers.

Salmeterol xinafoate is a potent, selective and long acting $\beta_{2}$ adrenoreceptor agonist used in the regular treatment of reversible airways obstruction $^{1}$ and available as a metered dose inhaler. Metered dose inhalers use chloro- fluorocarbons as propellants which face an international ban on production from 1995-6 due to their depleting effect on the ozone layer. Because of their "essential use" status, temporary exemptions have been granted for the use of chlorofluorocarbons in metered dose inhalers while satisfactory alternatives are developed. Salmeterol has been reformulated in Glaxo inhalation grade HFA134a (tetrafluoroethane).

Studies in animals have shown HFA134a to be a non-toxic, inert compound. It was well tolerated when given alone to healthy subjects in single and repeat doses up to a maximum of 10 actuations four times daily for 14 days. $^{2}$ HFA134a is rapidly absorbed following inhalation, and rapidly eliminated from the body by ventilation, with no evidence of accumulation or significant metabolism. ${ }^{3}$

We have investigated the safety and tolerability of the salmeterol/HFA134a inhaler in healthy subjects and compared its systemic pharmacodynamic activity with that of the current salmeterol inhaler using chlorofluorocarbon propellant 11 and 12 (P11/P12).

\section{Methods}

Twelve healthy men of mean age 26.9 years (range 19-40) and mean weight $76 \cdot 1 \mathrm{~kg}$ (range 59-92) gave their written informed consent. The study was approved by the local ethics committee and carried out in accordance with the provisions of the Declaration of Helsinki and later revisions.

Subjects received the salmeterol/HFA134a inhaler, the salmeterol/P11/12 inhaler, and placebo (HFA134a) in a double blind, randomised, three-way crossover manner on three different days at least one week apart. Salmeterol doses were cumulative: 50, 50, 100,

\footnotetext{
9 November 1994 Revised version received 10 January 1995 Accepted for publication 6 February 1995

Clinical

Division

Greenford

UK
} 